\title{
Article \\ Selective Desulfonylation of Oxidized Sulfur Compounds in Crude Oil by Alcohol-Mediated Nucleophilic Attack Studied by XANES and XRF Spectroscopy
}

\author{
Jonathan Rankin 1, Kyle Litz 1,*, Sarah Briggs 1, Trent McCaskill 1, Michael Scudder ${ }^{1}$, \\ Syed Khalid ${ }^{2}$ and Raman Budhani ${ }^{2}$ \\ 1 Auterra, Inc, 2135 Technology Drive, Schenectady, NY 12308, USA; jrankin@auterrainc.com (J.R.); \\ sbriggs@auterrainc.com (S.B.); tmccaskill@auterrainc.com (T.M.); mscudder@auterrainc.com (M.S.) \\ 2 Photon Sciences Directorate, 725 Brookhaven Ave., Brookhaven National Laboratory, \\ Upton, NY 11973, USA; Khalid@bnl.gov (S.K.); 09.raman@gmail.com (R.B.) \\ * Correspondence: klitz@auterrainc.com; Tel.: +1-518-382-9600; Fax: +1-518-382-9611
}

\begin{abstract}
Diesel, gas oil, whole crudes, and bitumen samples were subjected to sulfoxidation, and then subjected to hydroxide attack in the presence of ethylene or propylene glycol. The resultant oils were analyzed by XRF for total sulfur and XANES spectroscopy for sulfur speciation at each stage. The combination of these analyses gave the total amount of sulfur as organic sulfide, sulfoxide, and sulfone at each stage of treatment so as to determine the effectiveness of the desulfonylation for sulfur compounds of different oxidation states.
\end{abstract}

Keywords: desulfurization; desulfonylation; petroleum

\section{Introduction}

Due to regulations limiting the sulfur content allowed in many fuel oils, the challenges of deep hydrodesulfurization (HDS) have become more pressing. [1] Gasoline in the United States is planned to be regulated in 2017 to 10 ppm maximum by the U.S. Environmental Protection Agency [2], and marine fuels are regulated to $0.1 \%$ sulfur maximum in many areas as of 2015 . Reducing sulfur to levels necessary for meeting regulations requires increasing the temperature, pressure, and hydrogen consumption of the HDS treatment [3]. In addition, expensive catalysts are required for HDS treatment, which can be deactivated by contaminants in the feed, especially asphaltenes and metals like vanadium [4]. The U.S. Energy Information Administration reports that crude oil input to refineries is becoming heavier and more contaminated by heteroatoms such as sulfur $[5,6]$. The costs of HDS treatment thus continue to grow because of the required higher temperatures, excess hydrogen, and catalyst regeneration. Bitumen is especially difficult to process by these HDS methods [7], creating the need for development of a technology that would allow for more mild temperatures and pressures than current deep HDS technologies, and eliminate the need for expensive, sensitive catalysts. Bitumen is also more difficult to remove from the ground, having a higher viscosity than light crude oils, requiring more energy and thus $\mathrm{CO} 2$ emissions to extract. Present and future $\mathrm{CO} 2$ emission regulations also heighten the need for less energy-intensive means to treat these heavier crude oils.

Oxidative desulfurization (ODS) has appeal over HDS technologies due to the comparatively mild conditions used. ODS technologies can use relatively mild temperatures and pressures [8]. However, there are potential problems associated with the ODS approach. First, the ODS process must be selective to sulfur alone. Oxygenating hydrocarbons in the oil is undesirable and can reduce the overall value of the feed. Furthermore, once the sulfur compounds are oxidized to sulfones, the sulfur still must be removed from the oil. The sulfone molecules may be removed physically from the oil feed either by adsorption or extraction, but neither method is ideal because there is an inherent 
loss of value by removing the entire sulfur containing compound from the oil. For oil feeds with higher weight percentages of sulfur the yield loss by removing the sulfones can be substantial, and in the case of bitumen samples, the loss could be over $50 \%$.

Alkali metals like lithium or sodium can be used to convert organic sulfur compounds into hydrocarbons [9], but these reagents are far too expensive for regular commercial use. Some technologies attempt to utilize high temperature $\left(300-900^{\circ} \mathrm{C}\right)$ caustic leaching to convert the oxidized sulfur to sulfonates, sulfides, sulfite, or sulfate [10-12]. The high temperature technologies are normally only applied to coal or coke, since these processes typically create oxygenated compounds that would increase the viscosity and specific gravity of liquid oil, and thus lower the value. For example, the reaction of dibenzothiophene sulfone with a caustic like sodium hydroxide produces a high-density oxygenate, 2-hydroxybiphenyl, as the major product? ${ }^{7}$. Biological desulfurization has been attempted, but the process results in carbon oxidation, reducing the value of the oil [13].

The various methods of removing sulfur from oxidized sulfur compounds in complex matrices such as crude petroleum and bitumen heighten the need to understand product speciation both before and after desulfurization. X-ray Absorption Near Edge Structure (XANES) spectroscopy is an effective analytical method for determining the speciation of sulfur compounds in petroleum feedstocks, and quantifying the relative abundances of sulfides, thiophenes, sulfoxides, and sulfones [10,14-18].

It has been shown that the addition of various alcohols can provide control of the products of the reaction between dibenzothiophene sulfone and nucleophiles such as hydroxide [19]. The reaction of dibenzothiophene sulfone with ethylene glycol and hydroxide gave biphenyl as the major product, as shown in Figure 1. In the figure, E represents an electronegative group, and Nu represents a nucleophile. In the present study, a method of converting sulfur compounds in sulfoxidized petroleum feedstocks to non-oxygenated hydrocarbons is presented. The method produces oil products which have substantially lower sulfur and metals content, while properties like specific gravity and viscosity are improved relative to the starting material. The present method is shown to be equally useful for the treatment of petroleum distillates, whole crudes, and bitumen samples.

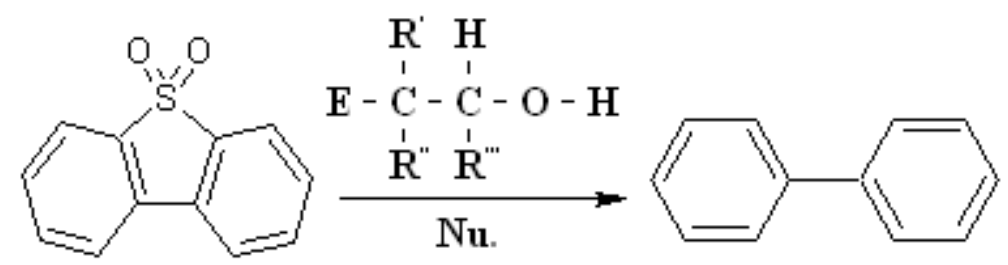

Figure 1. Conversion of Dibenzothiophene Sulfone to Biphenyl via Selective Desulfonylation.

\section{Materials and Methods}

Hydrogen peroxide, acetic acid, dibenzothiophene, diphenyl sulfide, dimethyl sulfoxide, dimethyl sulfone, sodium hydroxide, potassium hydroxide, and ethylene glycol were obtained from Sigma-Aldrich. Tert-butyl hydroperoxide, cumene hydroperoxide, and ethylbenzene hydroperoxide were obtained from Akzo Nobel. Samples of various petroleum distillates and whole crudes were obtained from suppliers in Canada, the United States, and Saudi Arabia.

The selective oxidation process has been described in detail elsewhere [19-23], and will be the subject of another separate publication. In general, a mixture of the oil to be treated and a peroxide was contacted with a heterogeneous catalyst, bis(glycerolato)oxotitanium(IV), at temperatures from $50-90^{\circ} \mathrm{C}$, with a residence time of 5-90 minutes. The mixture was continuously agitated, either by stirring or forced liquid flow. The following descriptions refer to the samples in Figure 1, 1a-1f. A $2.00 \%$ wt sulfur diesel (3a) sample from the United States Gulf Coast region and (3b) a sample of Athabasca bitumen containing $6.50 \%$ wt sulfur were treated continuously by the selective sulfoxidation process using bis(glycerolato)oxotitanium(IV) with hydrogen peroxide in acetic acid. 
For the diesel, some of the oil was extracted into the acid layer, and the two fractions were analyzed separately before being combined for desulfonylation.

In each case, the oil was recovered by distillation, and then treated with 3 molar equivalents to sulfur of mixed 1:1 sodium and potassium hydroxide and 2 molar equivalents to sulfur of ethylene glycol at $275^{\circ} \mathrm{C}$ for 60 minutes. The oil was washed with water to remove the base and glycol.

A sample of United States Gulf Coast Gas Oil containing 1.20\% wt sulfur, a sample of Arabian Whole Crude containing 3.15\% wt sulfur, and a sample of Athabasca Bitumen containing $4.34 \% \mathrm{wt}$ sulfur were treated by the selective sulfoxidation process using bis(glycerolato)oxotitanium(IV) with cumene hydroperoxide in cumene. The oils were separated by distillation, and then treated with 3 molar equivalents to sulfur of mixed 1:1 sodium and potassium hydroxide and 2 molar equivalents to sulfur of ethylene glycol at $275 \mathrm{C}$ for 60 minutes. The sample of Athabasca bitumen containing $4.82 \%$ wt sulfur was sulfoxidized using bis(glycerolato)oxotitanium(IV) and tert-butyl hydroperoxide. The oil was separated by distillation, then treated with 5 molar equivalents of phenol to sulfur, 6 molar equivalents of sodium hydroxide to sulfur, and 7 molar equivalents of propylene glycol to sulfur. In all cases, the oil was washed with water to remove the base and glycol.

A 1000 millilitre 316-stainless steel Parr high pressure reactor system with overhead stirring was used for all batch-wise desulfonylation experiments. Temperature was controlled by a 316-stainless steel thermocouple immersed in the liquid. The reactants used in the desulfonylation reaction include selectively sulfoxidized oil, a powdered base mix of $\mathrm{NaOH} / \mathrm{KOH}$ (1:1 by moles), and ethylene glycol. The nature of the feed oils tested includes diesel, gas oil, whole crude, and bitumen samples. To the cup of the $1000 \mathrm{~mL}$ Parr reactor was added the sulfoxidized oil and other reagents. The Parr reactor was sealed and placed in the furnace. The reactor was heated to $275^{\circ} \mathrm{C}$ and held for 60 minutes. The reactor was then cooled without stirring to room temperature. Oil samples were analyzed for percent weight sulfur, viscosity, and API gravity. If needed, the oils were centrifuged to remove residual caustic.

X-Ray Absorption Near Edge Structure (XANES) was used to determine the relative abundance of various sulfur oxidation states in untreated oils, selectively oxidized oils, and base/glycol treated oils. Raw spectra are available in the Electronic Supplementary Information. Combined with the total weight percent sulfur in the sample as measured by XRF, the weight percent of sulfide, sulfoxide, and sulfone can be determined. The XANES spectroscopy was carried out using beamline X-19A at the National Synchrotron Light Source (NSLS) at Brookhaven National Laboratory. The energy was varied from 2402 to $2572 \mathrm{eV}$, approximately $70 \mathrm{eV}$ below to $100 \mathrm{eV}$ above the sulfur K-shell absorption edge. Dibenzothiophene and diphenyl sulfide were used as standards for organic sulfides or thioethers, dimethyl sulfoxide was used as a standard for sulfoxides, and dimethyl sulfone and dibenzothiophene sulfone were used as standards for sulfones. These standards were used to determine the position of the sulfur K-shell absorption edge for each class of compounds. A software program called Athena was used to resolve the linear contribution of each class of compounds in petroleum samples to determine the relative abundance.

Viscosity was measured using a LVDV-I Prime Brookfield Viscometer. Viscosities were taken at $100^{\circ} \mathrm{C}$ using a hot oil circulator connected to the viscometer. The sulfur weight percent was determined by using an XOS Sindie 7039 XR, using monochromatic wavelength dispersive x-ray fluorescence (XRF). The measurement can be sensitive to water, solids, and oxygen present in the sample. The American Petroleum Institute (API) Gravity number was determined by using an appropriate floating hydrometer and a water bath to cool the oil to $15^{\circ} \mathrm{C}$. Nickel, vanadium, and iron content were measured by Adirondack Environmental Services in Albany, New York. Inductively coupled plasma optical emission spectrometry (ICP-OES) was used for quantification.

\section{Results}

\subsection{Sulfoxidation Catalyst}

The catalyst employed in the new selective oxidation process, bis(glycerolato)oxotitanium(IV), shown in Figure 2, rapidly oxidizes sulfur compounds commonly found in petroleum feedstocks 
without oxidizing olefins or benzylic hydrocarbons present in the oil [19-21]. The process demonstrates greater than $99 \%$ conversion of dibenzothiophene (DBT) to dibenzothiophene sulfone (DBTSO2) within 10 minutes at $85^{\circ} \mathrm{C}$ using ethylbenzene hydroperoxide, and greater than $99 \%$ conversion of 4,6-dimethyldibenzothiophene (DMDBT) to 4,6-dimethyldibenzothiophene sulfone (DMDBTSO2) within 10 minutes at $80^{\circ} \mathrm{C}$ using tert-butyl hydroperoxide. Meanwhile, no detectable oxidation of various olefins or benzylic hydrocarbons was detected within 80 minutes at the reaction temperature of $85^{\circ} \mathrm{C}$. The method has been discussed in detail elsewhere. [19-23]

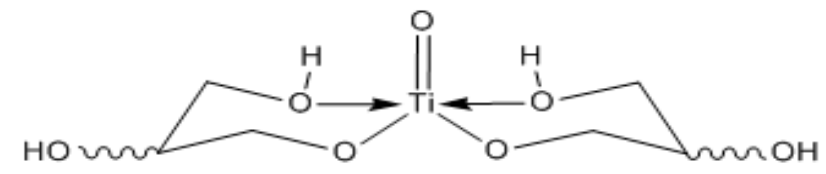

Figure 2. Structure of sulfoxidation catalyst bis(glycerolato)oxotitanium(IV).

\subsection{Petroleum Oils}

XRF analysis showed that in every case the sulfur content of the oil was reduced $35-70 \%$, typically near $50 \%$. To confirm the hypothesis that sulfones and sulfoxides in the oil were being converted to hydrocarbon compounds in a similar manner to dibenzothiophene sulfone, the products of these reactions were analyzed by XANES to determine sulfur speciation [10]. The results of the XANES and XRF analyses are combined in Figure 3. Each column shows the weight percent of the sulfur in the sample which is present in that particular oxidation state. Summing the three columns for a particular sample gives the total sulfur in the sample.

In general, the selective sulfoxidation procedure oxidized $40-70 \%$ of the organic sulfur in the oil to a mixture of sulfoxides and sulfones. As expected, the hydroxide and ethylene glycol treatment removed $70-100 \%$ of the sulfoxides formed, and $60-75 \%$ of the sulfones formed for the samples tested. The results were feed dependent, and likely affected by the different types of sulfur compounds in the feed. Organic sulfides which were not oxidized are expected to be inert to hydroxide attack, and were mostly unaffected by hydroxide and ethylene glycol treatment. Other polyols and aminealcohols were also evaluated in place of ethylene or propylene glycol and found to be generally effective [19], and will be the subject of future articles.

It is especially noteworthy that the two steps of this process performed similarly well on all samples, from petroleum distillates, to whole crudes, to bitumen samples. Most refining technology has increasing difficulty treating heavier oils, especially bitumen, which has high levels of sulfur, metals, and asphaltenes [7]. In addition to sulfur concentration, properties such as metals concentration, specific gravity, and viscosity are also important for the refining of petroleum oils, especially bitumen samples. Table 1 shows the initial and final values of these properties for the three bitumen samples tested. All samples showed a decrease in sulfur, metals, and viscosity at $100^{\circ} \mathrm{C}$, and an increase in API gravity at $15^{\circ} \mathrm{C}$, showing that the quality of the oil has improved, and suggesting that the sulfur in the oil was converted primarily to non-oxygenated hydrocarbons, which have lower densities and viscosities than their sulfur counterparts. 


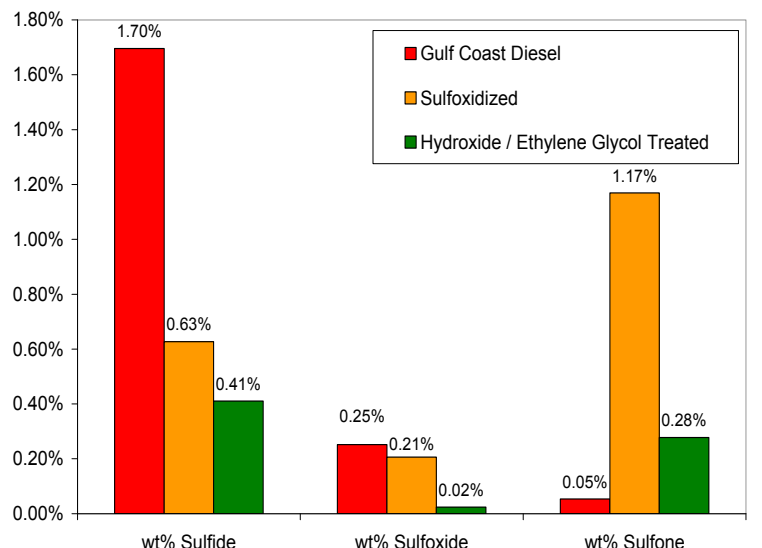

(a)

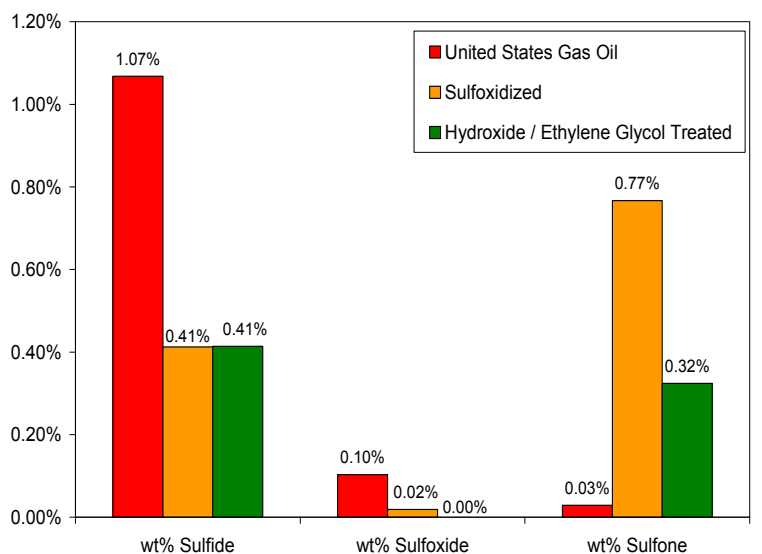

(c)

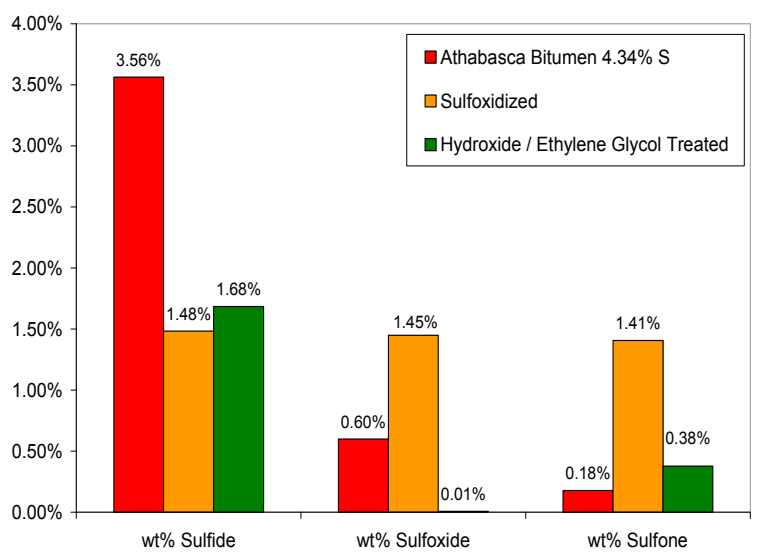

(e)

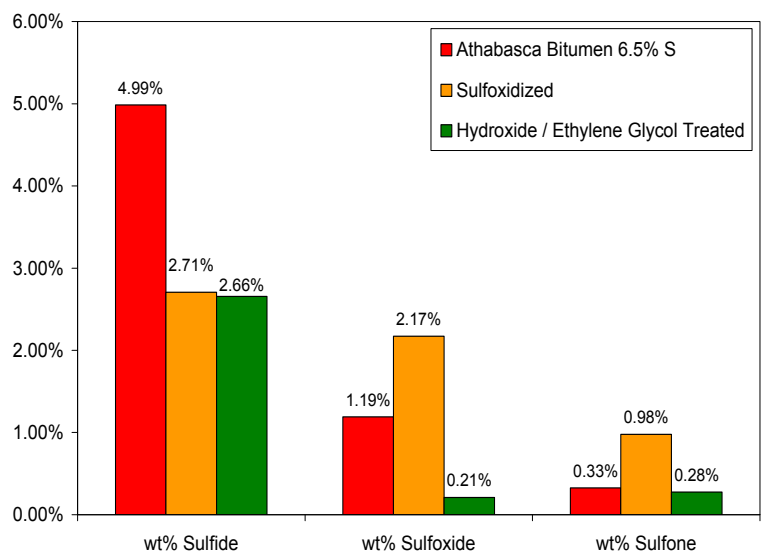

(b)

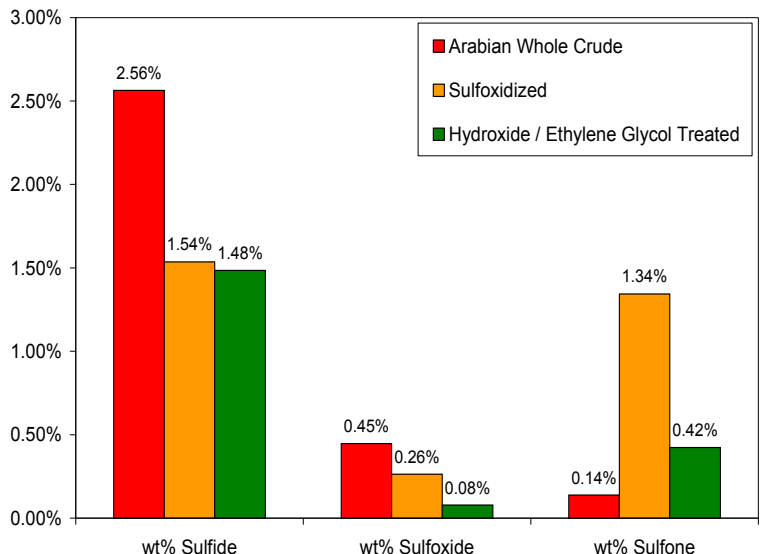

(d)

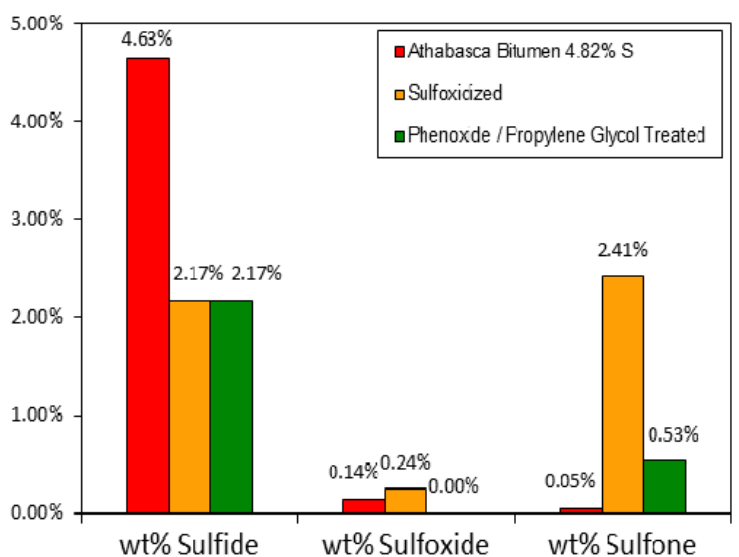

(f)

Figure 3. Results of Selective Sulfoxidation and Desulfonylation treatment of Petroleum Oils: (a) United States Gulf Coast Diesel, 2.00\% sulfur. (b) Athabasca Bitumen \#1, 6.50\% sulfur. (c) United States Gas Oil, 1.20\% sulfur. (d) Arabian Whole Crude, 3.15\% sulfur. (e) Athabasca Bitumen \#2, 4.34\% sulfur. (f) Athabasca Bitumen \#3, 4.82\% sulfur.

Figure 4 shows possible transformations of a hypothetical asphaltene molecule responsible for these changes. The asphaltene molecule (adapted from Speight [24] (p. 160), to give a reasonable sulfur content for an Athabasca asphaltene) shown in these sulfoxidation and desulfonylation reactions starts as $\mathrm{C}_{147} \mathrm{H}_{177} \mathrm{NSS}_{5}$, which becomes $\mathrm{C}_{147} \mathrm{H}_{177} \mathrm{NO}_{10} \mathrm{~S}_{5}$ after sulfoxidation, and finally breaks into several molecules as the C-S bonds are cleaved and replaced with hydrogen (shown for clarity), including $\mathrm{C}_{63} \mathrm{H}_{67} \mathrm{~N}, \mathrm{C}_{47} \mathrm{H}_{68}, \mathrm{C}_{25} \mathrm{H}_{38}$, and $\mathrm{C}_{10} \mathrm{H}_{14}$. These smaller molecules demonstrate the reasons for decreased density and viscosity of the treated oil. In addition, the boiling range of the oil would be 
expected to shift lower. At the time these experiments were performed, simulated distillation was not available. More recent experiments with bitumen analyzed by GC-SIMDIS have confirmed the shift in boiling points. See the electronic supplementary information for more detail. If the sulfoxidation process had oxidized carbons in the oil, such as benzylic or tertiary carbons, the density and viscosity of the oil would be expected to increase, which was not observed.

Table 1. Bitumen samples before and after treatment.

\begin{tabular}{|c|c|c|c|c|c|c|}
\hline Sample & $\begin{array}{l}\text { Sulfur } \\
(\text { wt } \%)\end{array}$ & $\begin{array}{l}\text { Iron } \\
\text { (ppm) }\end{array}$ & $\begin{array}{l}\text { Nickel } \\
\text { (ppm) }\end{array}$ & $\begin{array}{c}\text { Vanadium } \\
(\mathrm{ppm})\end{array}$ & $\begin{array}{c}\text { API Gravity } \\
\text { at } 15^{\circ} \mathrm{C}\end{array}$ & $\begin{array}{l}\text { Viscosity at } \\
100^{\circ} \mathrm{C} \text { (cP) }\end{array}$ \\
\hline Bitumen \#1 & $6.50 \%$ & 24 & 90 & 240 & $5.2^{\circ}$ & 700 \\
\hline Treated \#1 & $3.15 \%$ & 21 & 56 & 24 & $10.4^{\circ}$ & 425 \\
\hline Bitumen \#2 & $4.34 \%$ & 11 & 54 & 137 & $11.0^{\circ}$ & 180 \\
\hline Treated \#2 & $2.07 \%$ & 16 & 26 & 58 & $14.4^{\circ}$ & 152 \\
\hline Bitumen \#3 & $4.82 \%$ & 4 & 93 & 236 & $8.6^{\circ}$ & N/A \\
\hline Treated \#3 & $2.70 \%$ & 1 & 60 & 167 & $12.3^{\circ}$ & N/A \\
\hline
\end{tabular}
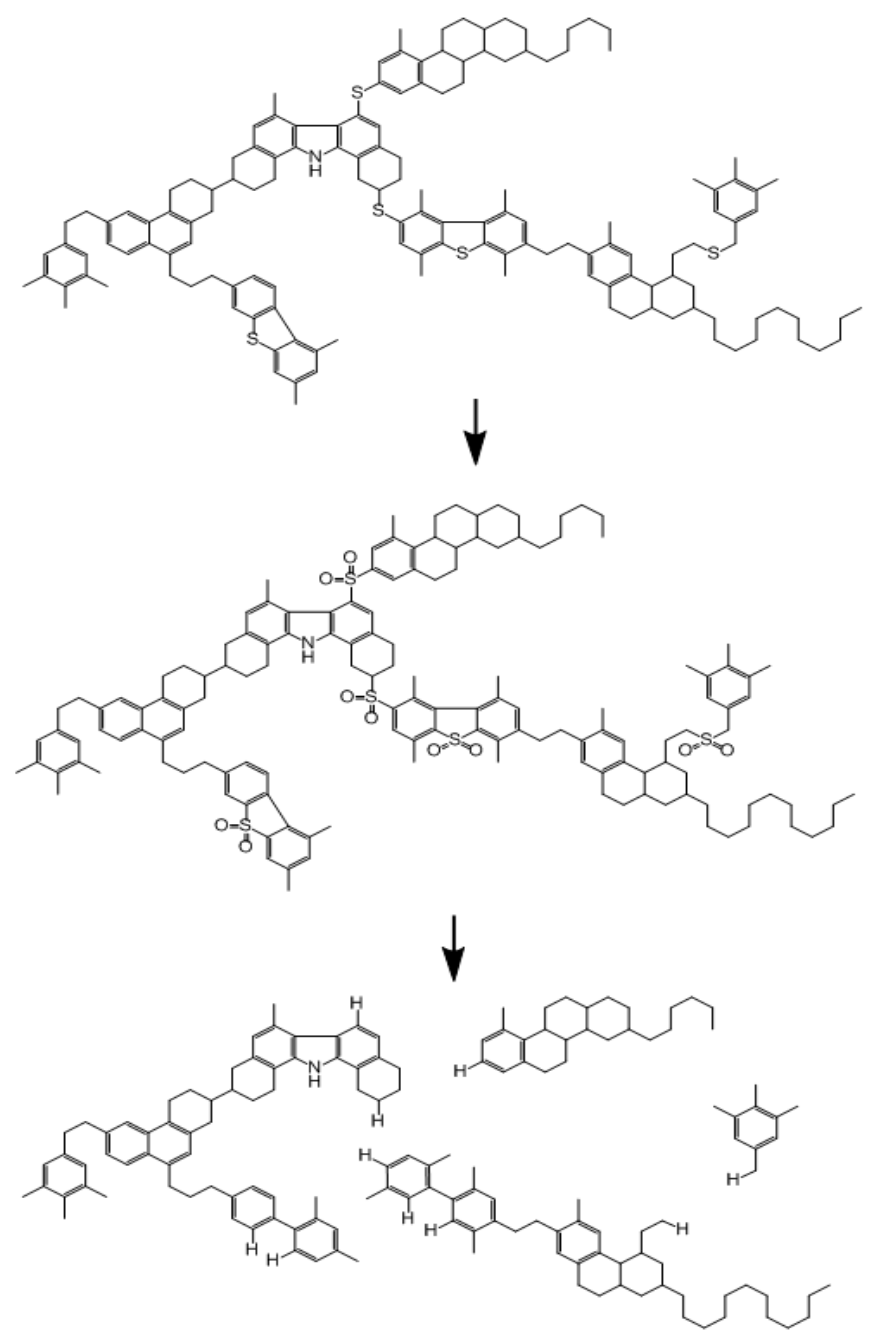

Figure 4. Representative molecular transformations in bitumen desulfonylation.

\section{Conclusions}

The desulfonylation reaction presented here shows promise for treating sulfoxidized petroleum feedstocks, since it produces a petroleum hydrocarbon as the product of the reaction. The new ODS treatment is equally effective in treating sulfoxidized petroleum feedstocks from diesel to bitumen 
while increasing the value of the oil, and is particularly effective against sulfoxides and sulfones in the oil.

Acknowledgments: The authors are grateful to Larry Lewis and Mark Banaszak Holl for help in editing this paper prior to publication. This research used beamline X19A of the National Synchrotron Light Source, a U.S. Department of Energy (DOE) Office of Science User Facility operated for the DOE Office of Science by Brookhaven National Laboratory under Contract No. DE-AC02-98CH10886.

Author Contributions: J.R. and K.L. conceived and designed the experiments; J.R., S.B., T.M., and M.S. performed the experiments; S.K. set up and maintained the XANES beamline, and R.B. analyzed the XANES data.

Conflicts of Interest: The authors declare no conflict of interest.

\section{References}

1. Song, C. An overview of new approaches to deep desulfurization for ultra-clean gasoline, diesel fuel and jet fuel. Catal. Today 2003, 86, 211-263.

2. Control of Air Pollution From Motor Vehicles: Tier 3 Motor Vehicle Emission and Fuel Standards; 2014; Vol. 79, pp. 23414-23886.

3. Landau, M. Deep hydrotreating of middle distillates from crude and shale oils. Catal. Today 1997, 36, $393-$ 429 .

4. Vogelaar, B. M.; Eijsbouts, S.; Bergwerff, J. A.; Heiszwolf, J. J. Hydroprocessing catalyst deactivation in commercial practice. Catal. Today 2010, 154, 256-263.

5. U.S. Sulfur Content (Weighted Average) of Crude Oil Input to Refineries http://www.eia.gov/dnav/pet/hist/LeafHandler.ashx?n=pet\&s=mcrs1us2\&f=a (accessed Mar 18, 2016).

6. U.S. API Gravity (Weighted Average) of Crude Oil Input to Refineries http://www.eia.gov/dnav/pet/hist/LeafHandler.ashx?n=pet\&s=mcrapus2\&f=a (accessed Mar 18, 2016).

7. Kim, J.; Longstaff, D.; Hanson, F. V Catalytic and thermal effects during hydrotreating of bitumen-derived heavy oils. Fuel 1998, 77, 1815-1823.

8. Lacount, R. B.; Friedman, S. Oxidation of Dibenzothiophene and Reaction of Dibenzothiophene 5,5-Dioxide with Aqueous Alkali. J. Org. Chem. 1977, 42, 2751-2754.

9. Morales, D. P.; Taylor, A. S.; Farmer, S. C. Desulfurization of dibenzothiophene and oxidized dibenzothiophene ring systems. Molecules 2010, 15, 1265-9.

10. Huffman, G.; Shah, N.; Huggins, F.; Stock, L. Sulfur speciation of desulfurized coals by XANES spectroscopy. Fuel 1995, 1094-1102.

11. George, Z. M.; Schneider, L. G.; Tollefson, E. L. Desulphurization of a fluid coke similar to the Athabasca oil sands coke. Fuel 1978, 57, 497-501.

12. Kocal, J. A.; Bricker, J. C.; Towler, G. P. Removal of sulfur-containing compounds from liquid hydrocarbon streams. US Pat, 7790021B2 2010.

13. Javadli, R.; de Klerk, A. Desulfurization of heavy oil. Appl. Petrochemical Res. 2012, 1, 3-19.

14. Kirtley, S. Sulfur species in Asphaltene, Resin, and Oil Fractions of Crude Oils by XANES and IR Spectroscopy Methods. APS Meet. Abstr. 2002.

15. Mansfield, C. T.; Barman, B. N.; Thomas, J. V.; Mehrotra, A. K.; McCann, J. M. Petroleum and Coal. Anal. Chem. 1999, 71, 81-108.

16. Mitra-Kirtley, S.; Mullins, O. C.; Ralston, C. Y.; Sellis, D.; Pareis, C. Determination of Sulfur Species in Asphaltene, Resin, and Oil Fractions of Crude Oils. Appl. Spectrosc. 1998, 52, 1522-1525.

17. Kasrai, M.; Bancroft, G. M.; Brunner, R.; Connan, J. The Chemical Nature of Oxidized Sulphur in Asphaltenes from X-Ray Absorption Spectroscopy. Le J. Phys. IV 1997, 7, C2-809-C2-810.

18. Gotte, V.; Rogalev, A.; Goulon, J.; Goulon-Ginet, C.; Michon, L.; Guilard, R.; Martin, D. A Study Using Sulfur K-Edge XAS of Bitumens, Asphaltenes, Maltenes and their Oxidation Products by Comparison with Model Compounds. Le J. Phys. IV 1997, 7, C2-667-C2-668.

19. Rankin, J. P.; Vreeland, J. L.; Litz, K. E.; Jordan, T. M.; Rossetti, M. N.; Burnett, E. H. Methods for upgrading of contaminated hydrocarbon streams. US Pat, 8197671B2 2012.

20. Amaratunga, G.; Dutta, P.; Jordan, T. M.; Lewis, S.; Litz, K. E.; Pawlson, J.; Rossetti, M.; Ullman, T.; Vreeland, J. M. Product containing monomer and polymers of titanyls and methods for making same. US Pat Appl, $20110119988 A 12011$. 
21. Litz, K. E.; Jordan, T. M.; Rosetti, M. N.; Loughran, A. J.; Vreeland, J. . Sulfoxidation Catalysts and Methods and Systems of Using Same. US Pat, 8394261B2 2013.

22. Litz, K. E.; Vreeland, J. L.; Rankin, J. P.; DeLancey, T. W.; Thompson, T. A. Reaction System and Products Therefrom. US Pat, 8298404B2 2012.

23. Litz, K. E.; Vreeland, J. L. Oxidative Desulfurization Using a Titanium(IV) Catalyst and Organohydroperoxides. US Pat, 8283498B2 2012.

24. Speight, J. G. The Desulfurization of Heavy Oils and Residua; Second Edi.; Marcel Dekker: New York, New York, 2000.

(C) 2016 by the authors; licensee Preprints, Basel, Switzerland. This article is an open access article distributed under the terms and conditions of the Creative Commons by

Attribution (CC-BY) license (http://creativecommons.org/licenses/by/4.0/). 